\title{
NF- $\kappa$ B and Nrf2 as prime molecular targets for chemoprevention and cytoprotection with anti-inflammatory and antioxidant phytochemicals
}

\author{
Young-Joon Surh $\cdot$ Hye-Kyung Na
}

Published online: 28 November 2007

(C) Springer-Verlag 2007

\section{Chemoprevention with dietary phytochemicals}

Cancer is a multifactorial heterogeneous disease characterized by multistage nature of pathogenesis. Over the past two or three decades, we have witnessed an enormous progress in the development of a vast variety of anticancer drugs and strategies. Nonetheless, we do not have magic bullets that can completely and selectively destroy malignant cells. Since neoplastic transformation, in general, is a relatively lengthy process that may take more than decades, there are ample opportunities to intervene in the pathogenesis of cancer, especially at early phases of oncogenesis. One such strategy is chemoprevention, an attempt to use either naturally occurring or synthetic substances, or their mixtures, to block, retard or even reverse the process of carcinogenesis. Numerous substances present in our daily diet, including fruits, vegetables, grains, spices, and seeds, have been shown to be effective in preventing cancer. Besides antioxidants, many plant-based organic chemical components, collectively called phytochemicals, retain pronounced chemopreventive potential. Currently, a series of human intervention trials are being considered with individual phytochemicals or their combination with known synthetic chemopreventive agents. However, precise assessment of underlying mechanisms of individual components is necessary before undertaking large-scale human trials.

The chemopreventive effects that most edible phytochemicals exert are likely to be the sum of several distinct

\footnotetext{
Y.-J. Surh $(\varangle) \cdot$ H.-K. Na
}

National Research Laboratory of Molecular Carcinogenesis and Chemoprevention, College of Pharmacy,

Seoul National University, Shinlim-dong,

Kwanak-ku, Seoul 151-742, Republic of Korea

e-mail: surh@plaza.snu.ac.kr mechanisms. These include blockage of metabolic activation and/or DNA binding of carcinogens, stimulation of detoxification, repair of DNA damage, suppression of cell proliferation and metastasis or angiogenesis, induction of differentiation or apoptosis of precancerous or maliganant cells, etc. [1]. Given the great structural diversity of phytochemicals, it is not feasible to define structure-activity relationships or any other commonalities to deduce their underlying molecular mechanisms. In this context, one of the promising approaches employed in studying the mechanisms of chemopreventive phytochemicals includes assessment of their effects on the specific components of signal transduction network that becomes often deregulated-either amplified or repressed-in many cancerous or transformed cells.

It has been known that a wide array of dietary phytochemicals act on the human genome, either directly or indirectly, to alter specific gene expression, thereby influencing the overall carcinogenic processes. Recently, much attention is being focussed on a new wave of nutrition research called "nutrigenomics". Nutrigenomics (or nutragenetics) can help understand how diseases such as cancer can be induced/aggravated or alleviated with dietary components by modulating specific gene expression. Nutrigenomic approaches are also applicable for the cancer chemoprevention studies.

Signal transduction pathway mediating inflammation and redox signaling:NF- $\kappa \mathrm{B}$ and $\mathrm{Nrf} 2$ as key player

In elucidating molecular mechanisms underlying chemopreventive or chemoprotective actions of dietary phytochemicals, components of signal transduction pathways have been often considered as potential targets. Since the 
cellular signaling network often goes awry in carcinogenesis, it is fairly rational and pragmatic to target intracellular signaling cascades for achieving chemoprevention [1]. Numerous molecules and events are involved in relaying intracellular signals. Both external and endogenous stimuli turn on or switch off critical events of this relay, thereby transmitting proper signaling to diverse downstream target molecules in a highly sophisticated fashion for fine-tuning of cellular homeostasis. Components of upstream or cytoplasmic signaling networks include protein kinases, such as the family of proline-directed serine/threonine kinases named mitogen-activated protein kinases (MAPKs), protein kinase $\mathrm{C}$ (PKC), phosphatidylinositol-3-kinase (PI3K), protein kinase $\mathrm{B} / \mathrm{Akt}$, glycogen synthase kinase, etc.

A new horizon in chemoprevention research is the recent discovery of molecular links between inflammation and cancer [2]. Modulation of cellular signaling involved in chronic inflammatory response by anti-inflammatory agents hence represents an important strategy in molecular target-based chemoprevention and cytoprotection [3]. Components of the cell signaling network, especially those which converge on the ubiquitous eukaryotic redox-sensitive transcription factor nuclear factor-kappaB (NF- $\kappa \mathrm{B})$, have been implicated in pathogenesis of many inflammation-associated disorders. Under normal physiologic conditions, NF- $\kappa \mathrm{B}$ is sequestered in the cytoplasm by binding to the inhibitory protein called $\mathrm{I} \kappa \mathrm{B} \alpha$. Phosphorylation and subsequent ubiquitination results in degradation of $\mathrm{I} \kappa \mathrm{B} \alpha$ by proteasomes (Fig. 1). Phosphorylation of $\mathrm{I} \kappa \mathrm{B} \alpha$ is mainly mediated by the $\mathrm{I} \kappa \mathrm{B}$ kinase (IKK) complex. Phosphorylation of specific serine residues of p65 subunit of NF- $\kappa \mathrm{B}$ has been considered to facilitate the translocation of NF- $\kappa \mathrm{B}$ to nucleus and interaction with the coactivator $\mathrm{CBP} / \mathrm{p} 300$.

Induction of phase-2 detoxifying or antioxidant genes represents an important cellular defence in response to oxidative and electrophilic insults. Nuclear transcription factor erythroid 2p45 (NF-E2)-related factor 2 (Nrf2) plays a crucial role in regulating phase-2 detoxifying/antioxidant gene induction. Like NF- $\kappa \mathrm{B}$, Nrf2 is present in the cytoplasm as an inactive complex with the inhibitory protein subunit, in this case Keap1. Dissociation of Nrf2 from Keap1 is prerequisite for nuclear translocation and subsequent transactivational activity. Once translocated into nucleus, Nrf2 interacts with a small Maf protein, forming a heterodimer that binds to antioxidant responsive elements (ARE) or electrophile responsive elements (EpRE) present in the promoter/enhancer regions of genes encoding many antioxidant and detoxifying enzymes (Fig. 2). Keap1 contains several cysteine residues that function as sensors of redox changes. Oxidation or covalent modification of these critical cysteine thiols diminishes the affinity of Nrf2 for Keap1, releasing Nrf2 for nuclear translocation.

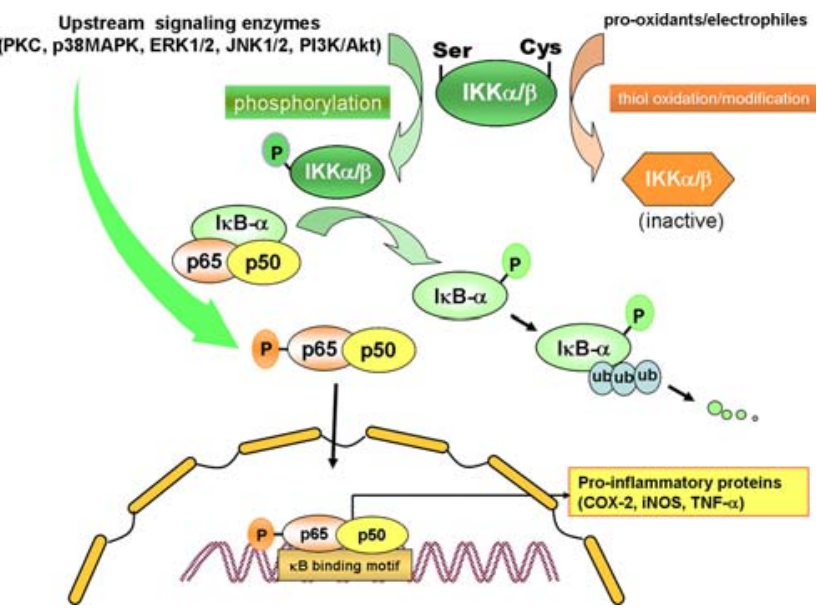

Fig. 1 Regulation of NF- $\kappa \mathrm{B}$ activation by cellular signaling molecules. Upon stimulation of cells, activation of protein kinases such as phosphatidylinositol 3-kinase (PI3K), protein kinase C (PKC), c-Jun $\mathrm{NH}_{2}$-terminal kinase (JNK), and extracellular signal-regulated kinase (ERK) induce phosphorylation of IKK $\alpha / \beta$. I $\kappa \mathrm{B} \alpha$ is rapidly phosphorylated by $\mathrm{IKK} \alpha / \beta$ and degraded via the ubiquitin-proteasome pathway. The resulting free NF- $\kappa \mathrm{B}$ dimer (p50-p65) translocates to the nucleus for the transcriptional regulation of multiple target genes. Some of the aforementioned proteine kinases may also catalyze the phosphorylation of p65 subunit of NF- $\kappa \mathrm{B}$, facilitating the nuclear translocation and/or interaction with the coactivator $\mathrm{CBP} / \mathrm{p} 300$. Chemopreventive phytochemicals can inhibit phosphorylation and/ or ubiquitination of $\mathrm{I} \kappa \mathrm{B} \alpha$ or phosphorylation of p65 induced by several oncogenic stimuli, thereby hampering the nuclear translocation and DNA binding of NF- $\kappa \mathrm{B}$. The cysteine residues present in IKK $\alpha / \beta$ are critical for catalytic activity and can be oxidized by prooxidants or covalent modified by electrophiles, leading to inactivation of the enzyme. Some dietary phytochemicals or their metabolites can act as prooxidants capable of directly oxidizing cysteine thiol or indirectly by altering cellular redox status and subsequently releasing ROS. Other groups of chemopreventive phytochemicals, especially those capable of acting as Michael reaction acceptors, are electrophilic per se and can hence directly modify the cysteine residues of IKK. Similar thiol modification by electrophilic phytochemicals was also found to occur for the p50 subunit of NF- $\kappa \mathrm{B}$ [30], but this event is not included in this illustration to avoid complexity

Dissociation of the Nrf2-Keap1 complex is also assumed to be stimulated through the phosphorylation of Nrf2 by distinct upstream kinases such as MAPKs, PKC, PI3K, etc. As in the case of NF- $\kappa \mathrm{B}$, phosphorylation of Nrf2 is also considered to facilitate the interaction of this redox-sensitive transcription factor with $\mathrm{CBP} / \mathrm{p} 300$. Many antioxidants derived from dietary and medicinal plants have been found to modulate Nrf2-Keap1 signaling, thereby potentiating cellular antioxidant capacity or facilitating detoxification of carcinogens and other toxicants $[4,5]$. It is noteworthy that there is a good correlation between anti-inflammatory activity of some chemopreventive/cytoprotective agents and their ability to induce antioxidant gene expression [6]. In this context, it is interesting to note that Nrf2 plays a role in protecting cells from not only oxidative stress but also 


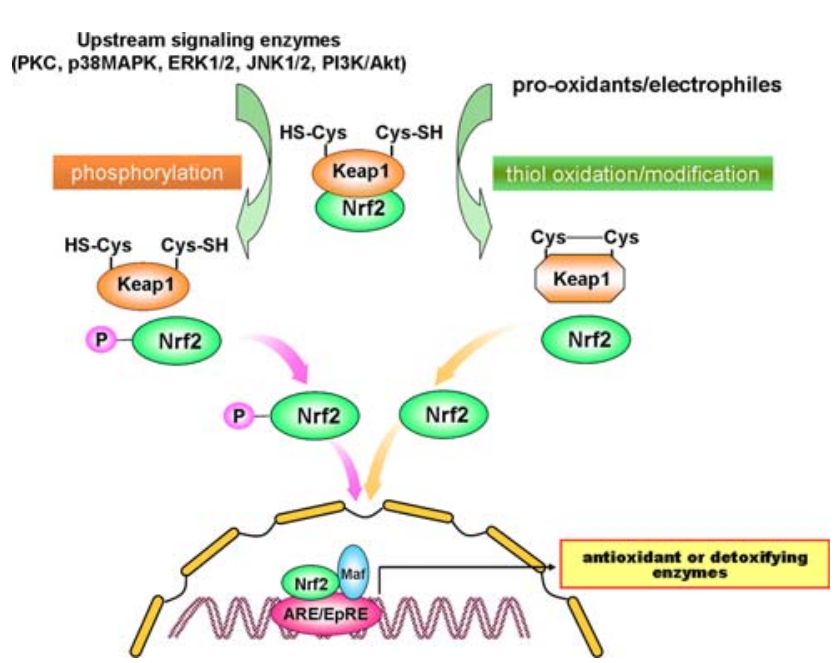

Fig. 2 Signal transduction pathways responsible for Nrf2-ARE activation. Nrf2 activation is regulated by two distinct cellular signaling pathways in a manner similar to that applied to NF- $\kappa \mathrm{B}$ activation signaling schematically represented in Fig. 1. Upon stimulation of cells, activation of protein kinases such as PI3K, PKC, JNK, p38MAPK and ERK induce Nrf2 phosphorylation, which facilitates the dissociation of Nrf2 from its repressor Keap1 and subsequent translocation to nucleus and interaction with the coactivator $\mathrm{CBP} / \mathrm{p} 300$. Alternatively, prooxidants or electrophiles may directly interact with cysteine residues present in Keap1, thereby stimulating Nrf2 dissociation. Both events can facilitate the nuclear translocation of $\mathrm{Nrf} 2$, which subsequently associates with small Maf, forming a heterodimer binds to antioxidant-response element (ARE) or electrophile-responsive element (EpRE) to stimulate phase II detoxification or antioxidant enzymes. Chemopreventive phytochemicals can activate Nrf2 signaling by inducing phosphorylation of Nrf2 via activation of the upstream kinases and/or through oxidation/ modification of Keap1 cysteine thiols

inflammatory insult [7-12]. The possible cross-talk between NF- $\kappa \mathrm{B}$ sinaling and that mediated by Nrf2 merits further investigation.

\section{Chemopreventive phytochemicals targeting NF- $\kappa$ B and Nrf2}

The current research in our laboratory concerns evaluation of various chemopreventive effects of some edible antioxidative and anti-inflammatory phytochemicals and elucidation of their underlying molecular mechanisms. Our research program has attempted to unravel common events mediated by redox-sensitive transcription factors such as $\mathrm{NF}-\kappa \mathrm{B}$ and $\mathrm{Nrf} 2$ and upstream kinases involved in the cellular signaling network for molecular target-based chemoprevention with selected dietary and medicinal phytochemicals, especially those with anti-inflammatory and/or antioxidative properties [1].

Curcumin, a yellow coloring agent contained in turmeric (Curcuma longa L., Zingiberaceae), has been reported to possess strong anti-tumor promotional as well as anti- inflammatory and antioxidant activities. Our recent studies have demonstrated that curcumin inhibits expression of cyclooxygenase-2 (COX-2) in mouse skin treated with the tumor promoter 12-O-tetradecanoylphorbol-13-acetate (TPA) through inactivation of the eukaryotic transcription factor NF- $\kappa \mathrm{B}$. Inhibition of NF- $\kappa \mathrm{B}$ by curcumin appears to be mediated by blocking ERK1/2 and p38 MAPK [13]. Oral administration of curcumin also inhibits azoxymethane-initiated and dextran sulfate sodium (DSS)-promoted colorectal carcinogenesis in mice (H.-S. Kim and Y.-J. Surh, manuscript in preparation).

[6]-Gingerol, a pungent ingredient present in ginger (Zingiber officinale Roscoe, Zingiberaceae), inhibited TPA-induced tumor necrosis factor-alpha production, ornithine decarboxylase activity and skin tumor promotion in female ICR mice [14]. Topically applied [6]-gingerol inhibited TPA-induced phosphorylation of p65 at Ser 536 and its interaction with the coactivator cAMP response element binding protein-binding protein $(\mathrm{CBP} / \mathrm{p} 300)$ in mouse skin, thereby rendering $\mathrm{NF}-\kappa \mathrm{B}$ transcriptionally inactive [15]. The NF- $\kappa \mathrm{B}$ inhibitory effects of [6]-gingerol appears to be associated with inhibition of p38 MAPK. [6]Gingerol also inhibited anchorage-independent growth of mouse epidermal JB-6 cells stimulated with epidermal growth factor [16]. More recently, [6]-gingerol has been shown to inhibit UVB-induced activation of NF- $\kappa \mathrm{B}$ and COX-2 expression in hairless mouse skin and also in an immortalized human keratinocytes cell line [17].

Capsaicin, a major pungent principle of hot chili pepper (Capsicum annuиm L., Solanaceae) with potential antiinflammatory and anti-tumor promoting properties, also suppressed TPA-induced activation of NF- $\kappa \mathrm{B}$ in mouse skin in vivo [18] as well as in cultured human promyelocytic leukemia HL-60 [19] and human myeloid ML-1a cells [20].

Resveratrol, a phytoalexin present in grapes and red wine, inhibited TPA-induced phosphorylation of $\mathrm{I} \kappa \mathrm{B} \alpha$ and subsequent p65 nuclear translocation in mouse skin by blocking IKK $\alpha$ and IKK $\beta$ [21]. A previous study from our laboratory has revealed that resveratrol rescues PC12 cells from oxidative stress via Nrf2-driven induction of heme oxygenase-1 (HO-1) expression [22].

The green tea polyphenol EGCG also inhibited activation of NF- $\kappa \mathrm{B}$ and AP-1 thereby suppressing the COX-2 induction in mouse skin in vivo and/or cultured human mammary epithelial (MCF10A) cells [23]. EGCG also upregulated antioxidant enzymes by activating the Nrf2ARE signaling pathway [24, 25].

Sulforaphane, an isothiocyanate present in cruciferous vegetables such as broccoli, has been extensively investigated with regards to its ability to induce phase 2 detoxification enzymes [26]. It activates Nrf2, possibly by modifying the sensor cysteines present in Keap1 [27, 28]. 


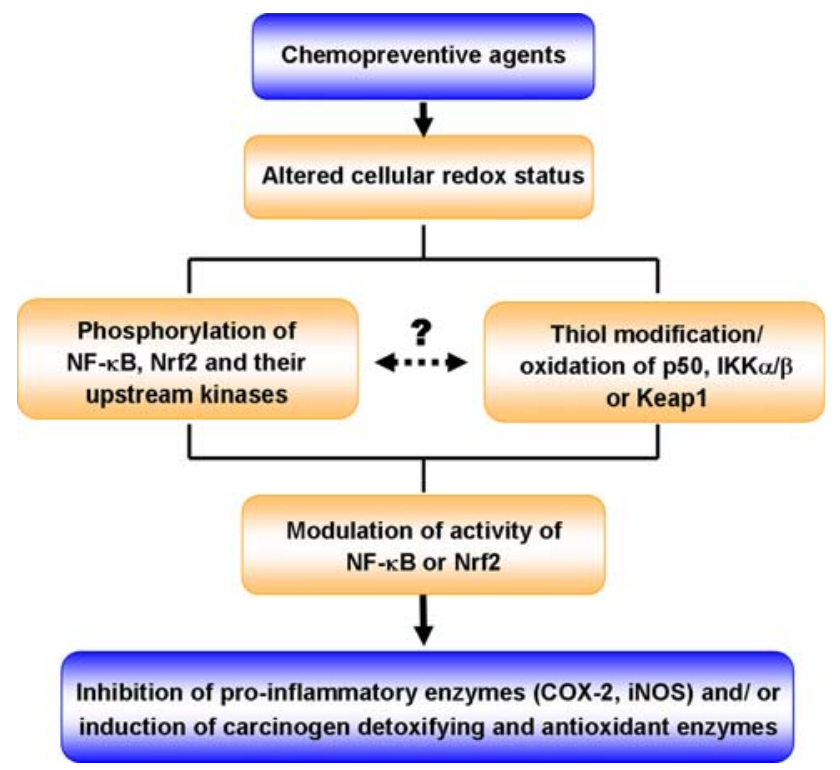

Fig. 3 Regulation of NF- $\kappa$ B and Nrf2-ARE signaling pathways by chemopreventive agents via phosphorylation and thiol modification/ oxidation of cellular signal molecules or their regulators

While considerable attention has focussed on sulforaphane as a "blocking" agent, with the ability to modulate Nrf2Keap1 signaling, it also exerts anti-inflammatory effects [29]. Thus, sulforaphane inhibited lipopolysaccharideinduced activation of NF- $\kappa \mathrm{B}$ and COX-2 expression in cultured mouse macrophages [30]. Interestingly, sulforaphane-induced inactivation of NF- $\kappa \mathrm{B}$ was associated with neither degradation of $\mathrm{I} \kappa \mathrm{B}$ nor nuclear translocation of NF$\kappa \mathrm{B}$, but rather attributable to its direct binding to essential thiol groups of p50, a functionally active subunit of NF- $\kappa \mathrm{B}$ subunits. In contrast to these findings, treatment of human mammary epithelial cells with sulforaphane inhibited TPAinduced COX-2 expression by blocking IKK activities and subsequent phosphorylation and degradation of $\mathrm{I} \kappa \mathrm{B} \alpha$, leading to suppression of NF- $\kappa \mathrm{B}$ activation [31]. Alternatively, sulforaphane may interact with reduced glutathione or other redox regulators like thioredoxin and Ref-1, resulting in perturbation of a reducing milieu relevant for the proper DNA binding of NF- $\kappa \mathrm{B}[32]$.

\section{Concluding remarks}

Oxidative stress and inflammatory tissue injuries are two of the most critical factors that are implicated in multistage carcinogenesis. Therefore, suppression of abnormally amplified inflammatory signaling or restoration/activation of improperly working or repressed antioxidant machinery can provide important strategies for chemoprevention. NF$\kappa \mathrm{B}$ and $\mathrm{Nrf} 2$ are major transcription factors that are involved in regulating proinflammatory and antioxidant genes, respectively. Many chemopreventive phytochemicals with anti-inflammatory activities inhibit NF- $\kappa \mathrm{B}$ activation via multiple mechanisms. Some antioxidative phytochemicals not only scavenge reactive oxygen species but also induces Nrf2-driven synthesis of antioxidant or phase 2 detoxification enzymes, thereby fortifying inherent cellular defence capacity against oxidative or electrophilic insults. Interestingly, majority of chemopreventive phytochemicals possess both anti-inflammatory and antioxidant properties. In consideration of close association between anti-inflammatory and antioxitant properties mediated by the same phytochemicals, it is worthwhile determining the cross-talk between NF- $\kappa \mathrm{B}$ and Nrf2 signaling.

Cysteine thiols present in various transcription factors (e.g., NF- $\kappa \mathrm{B}$ and Nrf2) and their regulators (IKK and Keap1) are recognized to function as redox sensors involved in fine-tuning of transcriptional regulation of many genes essential for maintaining cellular homeostasis (Fig. 3). Thus, oxidation or covalent modification of thiol groups present in redox-sensitive transcription factors and their regulating molecules can provide a unique strategy for molecular target-based chemoprevention and cytoprotection with anti-inflammatory and antioxidant phytochemicals [1].

Acknowledgments This work was supported by the research grant from the Korea Science and Engineering Foundation (KOSEF) for Biofoods Research Program, Ministry of Science Technology, Republic of Korea (awarded to Y.-J. Surh) and also by the grant (R082003-000-11081-0) from Korea Research Foundation (awarded to H.-K. Na).

\section{References}

1. Surh YJ (2003) Cancer chemoprevention with dietary phytochemicals. Nat Rev Cancer 3:768-780

2. Philip M, Rowley DA, Schreiber H (2004) Inflammation as a tumor promoter in cancer induction. Semin Cancer Biol 14:433439

3. Surh YJ, Kundu JK, Na HK, Lee JS (2005) Redox-sensitive transcription factors as prime targets for chemoprevention with anti-inflammatory and antioxidative phytochemicals. J Nutr 135:2993S-3001S

4. Lee JS, Surh YJ (2005) Nrf2 as a novel molecular target for chemoprevention. Cancer Lett 224:171-184

5. Yu X, Kensler T (2005) Nrf2 as a target for cancer chemoprevention. Mutat Res 591:93-102

6. Dinkova-Kostova AT, Holtzclaw WD, Wakabayashi N (2005) Keap1, the sensor for electrophiles and oxidants that regulates the phase 2 response, is a zinc metalloprotein. Biochemistry 44:6889-6899

7. Chen XL, Dodd G, Thomas S, Zhang X, Wasserman MA, Rovin BH, Kunsch C (2006) Activation of Nrf2/ARE pathway protects endothelial cells from oxidant injury and inhibits inflammatory gene expression. Am J Physiol Heart Circ Physiol 290:H1862H1870

8. Chen XL, Kunsch C (2004) Induction of cytoprotective genes through Nrf2/antioxidant response element pathway: a new 
therapeutic approach for the treatment of inflammatory diseases. Curr Pharm Des 10:879-891

9. Khor TO, Huang MT, Kwon KH, Chan JY, Reddy BS, Kong AN (2006) Nrf2-deficient mice have an increased susceptibility to dextran sulfate sodium-induced colitis. Cancer Res 66:1158011584

10. Li N, Nel AE (2006) Role of the Nrf2-mediated signaling pathway as a negative regulator of inflammation: implications for the impact of particulate pollutants on asthma. Antioxid Redox Signal 8:88-98

11. Rahman I, Biswas SK, Kirkham PA (2006) Regulation of inflammation and redox signaling by dietary polyphenols. Biochem Pharmacol 72:1439-1452

12. Yates MS, Kensler TW (2007) Chemopreventive promise of targeting the Nrf2 pathway. Drug News Perspect 20:109-117

13. Chun KS, Keum YS, Han SS, Song YS, Kim SH, Surh YJ (2003) Curcumin inhibits phorbol ester-induced expression of cyclooxygenase-2 in mouse skin through suppression of extracellular signal-regulated kinase activity and NF- $\kappa \mathrm{B}$ activation. Carcinogenesis 24:1515-1524

14. Park KK, Chun KS, Lee JM, Lee SS, Surh YJ (1998) Inhibitory effects of [6]-gingerol, a major pungent principle of ginger, on phorbol ester-induced inflammation, epidermal ornithine decarboxylase activity and skin tumor promotion in ICR mice. Cancer Lett 129:139-144

15. Kim SO, Kundu JK, Shin YK, Park JH, Cho MH, Kim TY, Surh YJ (2005) [6]-Gingerol inhibits COX-2 expression by blocking the activation of p38 MAP kinase and NF- $\kappa \mathrm{B}$ in phorbol esterstimulated mouse skin. Oncogene 24:2558-2567

16. Bode AM, Ma WY, Surh Y-J, Dong Z (2001) Inhibition of epidermal growth factor-induced cell transformation and activator protein 1 activation by [6]-gingerol. Cancer Res 61:850-853

17. Kim JK, Kim Y, Na KM, Surh YJ, Kim TY (2007) [6]-Gingerol prevents UVB-induced ROS production and COX-2 expression in vitro and in vivo. Free Radic Res 41:603-614

18. Han SS, Keum YS, Seo HJ, Chun KS, Lee SS, Surh YJ (2001) Capsaicin suppresses phorbol ester-induced activation of NF- $\kappa \mathrm{B} /$ Rel and AP-1 transcription factors in mouse epidermis. Cancer Lett 164:119-126

19. Han SS, Keum YS, Chun KS, Surh YJ (2002) Suppression of phorbol ester-induced NF- $\kappa$ B activation by capsaicin in cultured human promyelocytic leukemia cells. Arch Pharm Res 25:475479

20. Singh S, Nataraja K, Aggarwal BB (1996) Capsaicin (8-methyl$\mathrm{N}$-vanillyl-6-nonenamide) is a potent inhibitor of nuclear transcription factor $-\kappa \mathrm{B}$ activation by diverse agents. J Immunol $157: 4412-4420$
21. Kundu JK, Shin YK, Kim SH, Surh YJ (2006) Resveratrol inhibits phorbol ester-induced expression of COX-2 and activation of NF- $\kappa \mathrm{B}$ in mouse skin by blocking $\mathrm{I} \kappa \mathrm{B}$ kinase activity. Carcinogenesis 27:1465-1474

22. Chen CY, Jang JH, Li MH, Surh YJ (2005) Resveratrol upregulates heme oxygenase-1 expression via activation of NF-E2related factor 2 in PC12 cells. Biochem Biophys Res Commun 331:993-1000

23. Kundu JK, Na HK, Chun KS Kim YK, Lee SJ, Lee SS, Lee OS, Sim YC, Surh YJ (2003) Inhibition of phorbol ester-induced COX-2 expression by epigallocatechin gallate in mouse skin and cultured human mammary epithelial cells. J Nutr 133:3805S$3810 \mathrm{~S}$

24. We CC, Hsu MC, Hsieh CW, Lin JB, Lai PH, Wung BS (2006) Upregulation of heme oxygenase-1 by Epigallocatechin-3-gallate via the phosphatidylinositol 3-kinase/AkT and ERK pathways. Life Sci 78:2889-2897

25. Shen $\mathrm{G}, \mathrm{Xu} \mathrm{C}, \mathrm{Hu} \mathrm{R}$, Jain MR, Nair S, Lin W, Yang CS, Chan JY, Kong AN (2005) Comparison of (-)-epigallocatechin-3-gallate elicited liver and small intestine gene expression profiles between C57BL/6J mice and C57BL/6J/Nrf2 (-/-) mice. Pharmacol Res 22:1805-1820

26. Juge N, Mithen RF, Traka M (2007) Molecular basis for chemoprevention by sulforaphane: a comprehensive review. Cell Mol Life Sci 64:1105-1127

27. Dinkova-Kostova AT, Holtzclaw WD, Cole RN, Itoh K, Wakabayashi N, Katoh Y, Yamamoto M, Talalay P (2002) Direct evidence that sulfhydryl groups of Keap1 are the sensors regulating induction of phase 2 enzymes that protect against carcinogens and oxidants. Proc Natl Acad Sci USA 99:1190811913

28. Hong F, Freeman ML, Liebler DC (2005) Identification of sensor cysteines in human Keap1 modified by the cancer chemopreventive agent sulforaphane. Chem Res Toxicol 18:1917-1926

29. Myzak MC, Dashwood RH (2006) Chemoprotection by sulforaphane: keep one eye beyond Keap1. Cancer Lett 233:208-218

30. Heiss E, Herhaus C, Klimo K, Bartsch H, Gerhauser C (2001) Nuclear factor $\kappa \mathrm{B}$ is a molecular target for sulforaphane-mediated anti-inflammatory mechanisms. J Biol Chem 276:3200832015

31. Kim HN, Na HK, Kim EH, Surh YJ (2007) Proc Am Assoc Cancer Res

32. Heiss E, Gerhauser C (2005) Time-dependent modulation of thioredoxin reductase activity might contribute to sulforaphanemediated inhibition of NF- $\kappa$ B binding to DNA. Antioxid Redox Signal 7:1601-1611 